\title{
Identification of nucleic acid aptamers against lactate dehydrogenase via SELEX and high-throughput sequencing
}

\author{
Linghui Guo $^{1}$ - Ying Song ${ }^{1} \cdot$ Yanwen Yuan ${ }^{1}$ Jinlei Chen ${ }^{1} \cdot$ Haifeng Liang ${ }^{2} \cdot$ Fei Guo ${ }^{1} \cdot$ Zhi Yu $^{1} \cdot$ Pei Liang ${ }^{2}$. \\ Yapei Wang ${ }^{3} \cdot$ Pu Wang ${ }^{1}$
}

Received: 23 March 2021 / Revised: 21 April 2021 / Accepted: 7 May 2021 / Published online: 24 May 2021

(C) Springer-Verlag GmbH Germany, part of Springer Nature 2021

\begin{abstract}
Nucleic acid aptamers are small fragments of DNA or RNA molecules binding specifically to targets, which can be obtained through in vitro screening via systematic evolution of ligands by exponential enrichment (SELEX). Lactate dehydrogenase (LDH) is an important tumor marker, whose level in patients is of great significance for diagnosis of many diseases. Here, we report the identification of LDH aptamers by 9 rounds of screening from a length-mixed single-stranded DNA library using the SELEX technology. After the 3rd and 7th rounds of aptamer screening, affinity was significantly improved, and fluorescence quantitative analysis showed stronger affinity for the aptamers selected from the 7th to 9th rounds of screening. After highthroughput sequencing, motif analysis, and secondary structure prediction, we finally chose and further investigated 15 candidate LDH aptamer sequences with obvious differences in secondary structure in the 7th to 9th rounds of screening. Among them, LDH7-1, LDH7-9, LDH8-2, and LDH9-1 were shown to bind to LDH protein with high affinity and specificity with $K_{d}<25 \mathrm{nM}$. This study provides new ideas for rapid detection of LDH protein content and enzyme activity, thus contributing to the development of rapid medical detection.
\end{abstract}

Keywords SELEX $\cdot$ Lactate dehydrogenase $\cdot$ Nucleic acid aptamers $\cdot$ High-throughput sequencing

\section{Introduction}

Compared with normal cells, malignant tumor cells are characterized biochemically by their high glycolytic activity and the ability to produce large amounts of lactic acid [1]. Lactate dehydrogenase (LDH), a key enzyme in the glycolysis process, catalyzes the production of lactic acid during the

Yapei Wang

xiaomi93@163.com

$\triangle \mathrm{Pu}$ Wang

pwang@mail.hzau.edu.cn

1 Key Laboratory of Horticultural Plant Biology, Ministry of Education, College of Horticulture and Forestry Sciences, Huazhong Agricultural University, Wuhan 430070, Hubei, China

2 College of Optical and Electronic Technology, China Jiliang University, Hangzhou 310018, Zhejiang, China

3 Department of Gynecology and Obstetrics, The Central Hospital of Wuhan, Tongji Medical College, Huazhong University of Science and Technology, Wuhan 430014, Hubei, China reduction reaction of the pyrrole ring, coupled with the mutual conversion of NADH and $\mathrm{NAD}^{+}$. This enzyme exists in human tissues in the form of 5 independent isoenzymes, with each isoenzyme consisting of two subunits $\mathrm{A}$ and $\mathrm{B}$, which are respectively composed of a tetramer encoded by independent genes [2]. LDH exists in almost all types of cells in the human body, most commonly in myocardium, skeletal muscle, and red blood cells, and LDH varies in its form in different tissues, with LDH-1 in cardiomyocytes, LDH-2 in the reticular endothelial system, LDH-3 in the lungs, LDH-4 in the kidneys and plasma, and LDH-5 in the liver and striated muscle [3]. Meanwhile, LDH is a cancer detection marker, whose level in patient serum, cultured cells, and tumor tissues is of great significance for early diagnosis of tumors [4]. Determining the LDH level in patients can help the diagnosis of various types of cancers, such as oral cancer [5], stomach cancer [6], lung cancer [7], cervical cancer [8], or breast cancer [9], because patients with such cancers usually show an increase in the LDH expression level relative to healthy people, which is a sign of poor tumor prognosis. LDH is not only a key enzyme 
involved in the metabolism of cancer cells, but also can make tumor cells escape or suppress the immune system by changing the tumor microenvironment [10]. Additionally, statistics of the new type of coronavirus (COVID-19) revealed a certain correlation between the increase of LDH levels in patients and the poor prognosis of the disease process, i.e., an increase in the LDH level in COVID-19 patients suggests a 6-fold increase in disease severity and a 16-fold increase in mortality risk [11]. Among pediatric patients, LDH level is higher in girls than in boys [12].

LDH detection is widely used in clinical medicine, and its detection efficiency can be enhanced by using nucleic acid aptamers to improve the existing detection technology. Nucleic acid aptamers are small fragments of singlestranded DNA or RNA molecule that can bind to the target substance with high specificity and affinity, and they can be obtained by in vitro screening of artificially synthesized oligonucleotide libraries through systematic evolution of ligands by exponential enrichment (SELEX) [13]. There are a wide variety of target substances for nucleic acid aptamers, such as proteins, living cells, microorganisms, viruses, small biological molecules, and even tissues [14]. Nucleic acid aptamers are also called "chemical antibodies," but when compared with antibodies, aptamers can be obtained through in vitro screening without animal experiments and have the advantages of a shorter production cycle, labeling and modification by chemical synthesis, higher stability, and longer storage time. Previous studies have shown that the dissociation constant $\left(K_{d}\right)$ of the binding of nucleic acid aptamers to the target substance can reach the nanomolar or even picomolar level, with the specific antibody-antigen binding affinity being equivalent or even higher than that of antibody [15]. In the past few years, great progress has been in the SELEX technology, leading to the emergence of Cell-SELEX, Tissue-SELEX, and other technologies, greatly increasing the target materials of aptamers, and facilitating the application of aptamers in the medical field [16-19]. Meanwhile, aptamer screening has been greatly improved by combining microfluidic chip technology with SELEX technology, and aptamer screening for multiple targets can be completed in just a few hours [20,21]. Nucleic acid aptamers not only have a good application prospect in diagnosis of tumors and diseases, but also can be used in combination with a variety of drugs or nanomaterials for targeted therapy of tumors to improve drug delivery efficiency, reduce toxicity to normal cells, alleviate stress, assuage side effects, and improve the quality of life of cancer patients [22, 23].

Currently, the colorimetric method is the main method of quantitative detection of LDH enzyme activity, lacking of protein content quantification. In the catalyzation process of lactate dehydrogenase, NAD+ is reduced to NADH.
Generated NADH and INT (2-p-iodophenyl-3-nitrophenyl tetrazolium chloride) are catalyzed into a strong chromophore (formazan) by diaphorase. Formazan has an absorption peak at a wavelength of $490 \mathrm{~nm}$, which is used to determine the activity of lactate dehydrogenase [24]. However, the colorimetric method still has many shortcomings. Human serum is a mixture of various proteins, leading to interference of $\mathrm{LDH}$ enzyme activity by other substances. Besides, the colorimetric method is seriously affected by the reaction time and temperature, which causes the inaccuracy on the quantitative detection of LDH enzyme activity. In this study, 9 rounds of aptamer screening were carried out, and 4 aptamer sequences with high affinity and specificity to LDH protein were obtained. This study provides new ideas for rapid detection of tumor markers and shows the possibility for home detection of disease serum markers, thus promoting the development of rapid medical detection.

\section{Materials and methods}

\section{Materials}

Carboxyl-activated magnetic beads were obtained from Changzhou Smart-lifesciences Biotechnology Co., Ltd, (Guangzhou, China); pure lactate dehydrogenase (L-LDH) and normal human serum from Solarbio Life Sciences (Beijing, China); alpha fetoprotein (AFP) from National Institutes for Food and Drug Control (Beijing, China); bovine serum albumin (BSA) from Biofroxx (Germany); PageRuler prefabricated protein ladder from ThermoFisher Scientific (Waltham, MA, USA); DNA purification kit and gel DNA recovery Kit from Simgen (China); $2 \times$ Taq PCR Mix from Aidlab Biotechnologies Co., Ltd. (Beijing, China).

The common primers used for aptamer screening and PCR, and the forward and reverse primers labeled with Texas Red or FAM (5'-ATAGGAGTCACGACGACCAGAA-3'; 5'ATTAGTCAAGAGGTAGACGCACATA-3') were all synthesized by Beijing Tsingke Biological Technology Co., Ltd. (Beijing, China), and purified by HPLC. The other reagents used in this experiment were of analytical grade, and the water used was ultrapure water.

\section{Length-mixed ssDNA library}

A random single-stranded DNA (ssDNA) library was constructed with lengths of $77 \mathrm{nt}, 87 \mathrm{nt}, 97 \mathrm{nt}$, and $107 \mathrm{nt}$ and with random sequences of $30,40,50$, and 60 bases in the middle (Table 1).

The ssDNA libraries of the above four lengths were dissolved in dd $\mathrm{H}_{2} \mathrm{O}(10 \mu \mathrm{M})$ and mixed in equal volumes to form a length-mixed ssDNA library. 
Table 1 Random ssDNA library and primers

\begin{tabular}{ll}
\hline ssDNA library & Sequences $\left(5^{\prime}-3^{\prime}\right)$ \\
\hline N30 & ATAGGAGTCACGACGACCAGAA-N ${ }_{30}$-TATGTGCGTCTACCTCTTGACTAAT \\
N40 & ATAGGAGTCACGACGACCAGAA-N 40 -TATGTGCGTCTACCTCTTGACTAAT \\
N50 & ATAGGAGTCACGACGACCAGAA-N 50 -TATGTGCGTCTACCTCTTGACTAAT \\
N60 & ATAGGAGTCACGACGACCAGAA-N 6 -TATGTGCGTCTACCTCTTGACTAAT \\
\hline
\end{tabular}

\section{Coupling of LDH protein to carboxyl magnetic beads}

Carboxyl-activated Magpoly Beads (carboxyl magnetic beads) were used to bind the LDH protein. Briefly, carboxyl magnetic beads $\left(5 \times 10^{7}\right.$ in $\left.50 \mu \mathrm{L}\right)$ were well mixed, followed by discarding the protective solution, adding activation solution $(50 \mathrm{mM}$ MES, $\mathrm{pH}=5.0)$ to activate the magnetic beads. The activated magnetic beads were incubated with $1 \mathrm{mg} / \mathrm{mL} \mathrm{LDH}$ protein dissolved in the activation solution overnight on a rotation mixer (Qilinbeier WH-986, Kylin-Bell Lab Instruments Co., Ltd., China) at room temperature for sufficient coupling. After incubation, the supernatant was collected, followed by adding $125 \mu \mathrm{L}$ blocking solution (50 mM Tris, $\mathrm{pH} 7.4)$ to the magnetic beads to block their surface sites. Then, nonspecific adsorption was removed by washing with $0.1 \%$ Tween20 in $1 \times$ PBS (pH7.4) twice and $1 \times$ PBS twice. Besides, the coupling processes of BSA, normal human serum, and AFP to carboxyl magnetic beads were the same.

\section{SDS-PAGE analysis}

The coupling effect between magnetic beads and protein was determined by SDS-PAGE analysis. Briefly, $5 \times$ protein loading buffer was added to the protein-coupled magnetic beads and the supernatant, followed by a warm water bath at $95{ }^{\circ} \mathrm{C}$ for $15 \mathrm{~min}$. After cooling to room temperature, the coupling effect between the magnetic beads and protein was evaluated by $12 \%$ SDS-PAGE electrophoresis. The gel was stained with $0.1 \%$ Coomassie Brilliant Blue R-250 for $2 \mathrm{~h}$ and destained overnight on a rocking platform.

\section{SELEX}

The short-stranded salmon sperm DNA $(5 \mu \mathrm{M}, 200 \mu \mathrm{L})$ [25] and the length-mixed ssDNA library (the concentration of each length-specific ssDNA was $2.5 \mu \mathrm{M}, 200 \mu \mathrm{L}$ ) were denatured at $95{ }^{\circ} \mathrm{C}$ for $8 \mathrm{~min}$ and cooled in an ice bath for $8 \mathrm{~min}$, followed by adding salmon sperm DNA to the carboxyl magnetic beads $\left(5 \times 10^{7}\right.$ in $\left.50 \mu \mathrm{L}\right)$ coupled with $\mathrm{LDH}$ protein $(1 \mathrm{mg} / \mathrm{mL}, 5 \mu \mathrm{L})$, and adding the mixed ssDNA library to the carboxyl magnetic beads coupled with normal human serum $\left(5 \times 10^{7}\right.$ in $\left.50 \mu \mathrm{L}\right)$. Next, the two mixtures were supplemented with $400 \mu \mathrm{L}$ binding buffer $(50 \mathrm{mM}$ Tris- $\mathrm{HCl}, 5 \mathrm{mM} \mathrm{KCl}, 100 \mathrm{mM} \mathrm{NaCl}$, $1 \mathrm{mM} \mathrm{MgCl} 2, \mathrm{pH} \mathrm{7.4)}$ and incubated for $45 \mathrm{~min}$ at room temperature on a rotation mixer. After incubation, the supernatant of the LDH protein system was discarded, and the magnetic beads were washed twice with binding buffer, followed by adding the supernatant of the normal human serum system to the magnetic beads of the LDH protein system and incubation at room temperature for 45 min. After discarding the supernatant, the magnetic beads were washed twice with binding buffer, followed by adding $100 \mu \mathrm{L}$ dd $\mathrm{H}_{2} \mathrm{O}$, a water bath at $95{ }^{\circ} \mathrm{C}$ for $8 \mathrm{~min}$, and then an ice bath for $8 \mathrm{~min}$ to separate DNA and magnetic beads. The supernatant was the selected aptamer sequence and used as a template for PCR amplification. The PCR was performed under the conditions of $94{ }^{\circ} \mathrm{C}$ pre-denaturation for $5 \mathrm{~min} ; 30$ cycles of $95{ }^{\circ} \mathrm{C}$ denaturation for $45 \mathrm{~s}, 60{ }^{\circ} \mathrm{C}$ annealing for $15 \mathrm{~s}$, and $72{ }^{\circ} \mathrm{C}$ extension for $15 \mathrm{~s}$; and $72{ }^{\circ} \mathrm{C}$ final extension for $1 \mathrm{~min}$. The PCR product was purified and used for the next round of screening, and a total of 9 rounds of aptamer screening were performed.

\section{Fluorescence microscopy imaging}

Changes in the affinity of the aptamers from the 1-9 rounds of screening and the affinity and negative screening effect of 15 selected candidate LDH aptamer sequences were evaluated using an inverted fluorescence microscope (Leica DMi8, Leica Microsystems, Germany). Briefly, the aptamer library was amplified by PCR with primers labeled with Texas Red. Next, the labeled aptamer library was incubated with the magnetic beads coupled with LDH protein, normal human serum, AFP, or BSA overnight at room temperature, followed by discarding the supernatant, washing the magnetic beads twice with binding buffer. Finally, images were captured using the aforementioned inverted fluorescence microscope.

\section{Fluorescence intensity measurement}

Briefly, the Texas Red-labeled aptamer library with stronger affinity (after 7-9 rounds of screening) was incubated 
with the magnetic beads coupled with LDH protein overnight at room temperature. After incubation, the supernatant was discarded, and the magnetic beads were washed twice with binding buffer, followed by adding $20 \mu \mathrm{L}$ dd $\mathrm{H}_{2} \mathrm{O}$, a water bath at $95{ }^{\circ} \mathrm{C}$ for $8 \mathrm{~min}$, and then an ice bath for $8 \mathrm{~min}$. After cooling, the magnetic beads were discarded and the supernatant was collected. Finally, the fluorescence intensity was measured using a fluorescence spectrophotometer (Nanodrop 3300, Thermo Fisher Scientific, USA) and each measurement was repeated three times.

\section{High-throughput sequencing and bioinformatics analysis}

After PCR amplification of the aptamers obtained from the 9 rounds of screening, the PCR products were purified and sent to Novogene Co. Ltd. (Beijing, China) for library construction and high-throughput sequencing (HTS) using the Illumina Platform. Briefly, the adaptors were added into the DNA pool and PCR amplification was carried out. DNA purification was performed by AMPure XP beads and the insert size was examined by Agilent 2100 bioanalyzer. After the library quality was qualified, it was sequenced on the computer. The sequencing data were analyzed using a self-compiled program, and the results were submitted to the MEME website [26] to analyze the conserved elements. Finally, the secondary structure and free energy of the aptamers were predicted using NUPACK (http://www.nupack.org/) [27].

\section{Dissociation constant $\left(K_{d}\right)$ measurement}

The dissociation constant $\left(K_{d}\right)$ of the candidate aptamer was measured by the method of fluorescence assay to evaluate the affinity of the selected candidate LDH aptamer sequences [28]. In detail, we diluted the FAMlabeled aptamer candidate sequences and the ssDNA library to gradient concentrations $(10 \mathrm{nM}, 20 \mathrm{nM}, 40 \mathrm{nM}$, $80 \mathrm{nM}, 160 \mathrm{nM}$, and $320 \mathrm{nM}$ ), and combined these dilutions with carboxyl magnetic beads coupled with LDH protein $\left(5 \times 10^{7}\right)$, incubating overnight at room temperature on a rotation mixer. After the incubation, the supernatant was discarded, and the magnetic beads were washed twice with binding buffer. Then, $20 \mu \mathrm{L}$ dd $\mathrm{H}_{2} \mathrm{O}$ was added, followed by a 8 -min water bath at $95{ }^{\circ} \mathrm{C}$ and a 8 -min ice bath. The amount of released aptamers in supernatant was measured by a fluorescence assay at $520 \mathrm{~nm}$. The fluorescence intensity results were fit by the kinetic curve and the dissociation constant $\left(K_{d}\right)$ was calculated. The measurement of fluorescence intensity for each sample was repeated 3 times.

\section{Statistical analysis}

In this study, an average of 10 fields of view was included in the statistical analysis of all microscope data, with at least 15 magnetic bead groups in each field. The analysis of variance (ANOVA) was performed using GraphPad Prism 8 software. Each set of experiments was performed with three biological replicates and three technical replicates.

\section{Results and discussion}

\section{SELEX screening of ssDNA LDH aptamers}

For SELEX screening of ssDNA LDH aptamers, the coupling effect between LDH protein and carboxyl magnetic beads was evaluated by SDS-PAGE analysis. As shown in Fig. 1a, lanes 1 and 2 showed that there was no LDH protein in normal human serum. Besides, there was obvious LDH protein band in lane 3 , and the presence of LDH protein band in lane 4 indicated that LDH protein can be coupled with carboxyl magnetic beads. The SELEX screening process is shown in Fig. $1 \mathrm{~b}$. Specifically, in each round, the ssDNA library was incubated with normal human serum as a negative screening, while small fragments of salmon sperm DNA were incubated with magnetic bead-coupled LDH protein to block the non-specific binding sites. Next, the ssDNA unbound to normal human serum was incubated with the magnetic beads coupled with LDH protein to obtain the ssDNA specifically binding to LDH protein. Finally, the ssDNA was dissociated and used as a template for PCR amplification, and the recovered PCR product was used for HTS sequencing and the next round of screening. Negative screening with normal human serum as the target can pre-clear aptamers in the ssDNA library that can bind to other targets in normal human serum and improve the specificity of the library in the subsequent screening [29]. In the case of fixed LDH protein content, blocking non-specific binding sites by salmon sperm DNA can reduce the number of binding sites between LDH protein and ssDNA library, thereby inhibiting the enrichment of low-affinity aptamers and only retaining high-affinity aptamers [30]. In order to increase the diversity of the initial ssDNA library to obtain aptamer sequences with stronger affinity and specificity with the LDH protein, we constructed a special library with four sub-libraries of different lengths (Fig. 1c). In order to obtain more comprehensive and true information about aptamers and compare their differences between rounds, we used HTS sequencing to analyze the aptamers obtained in each round. 

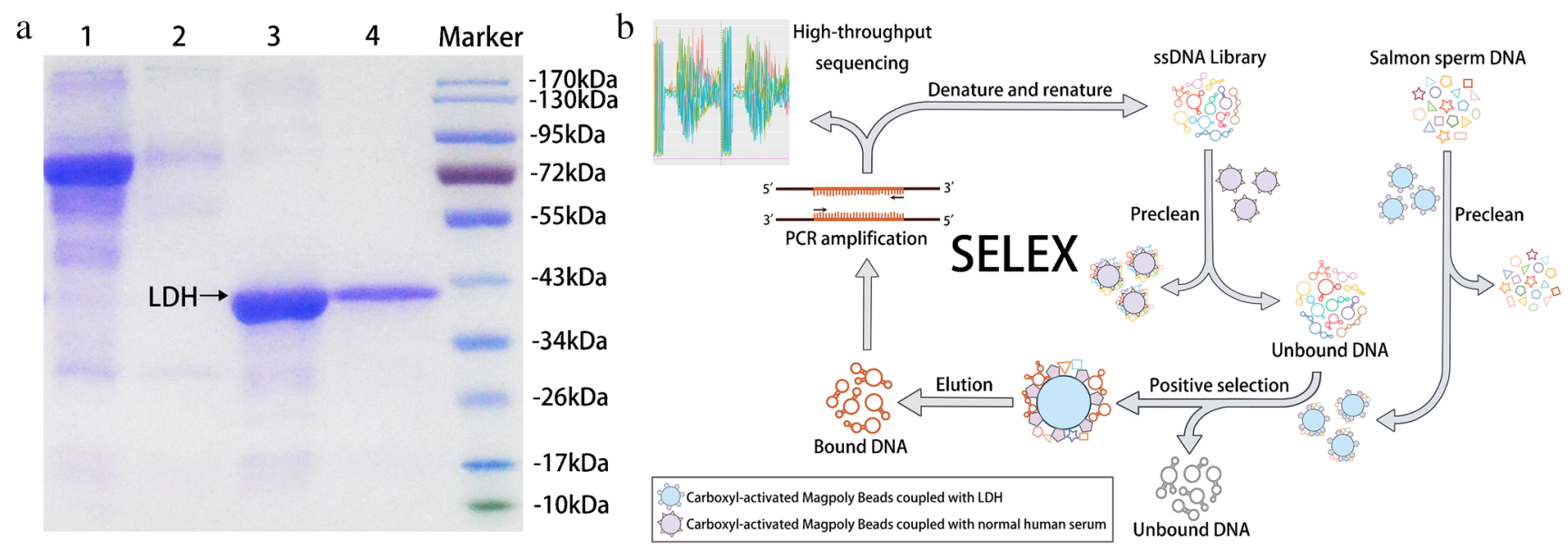

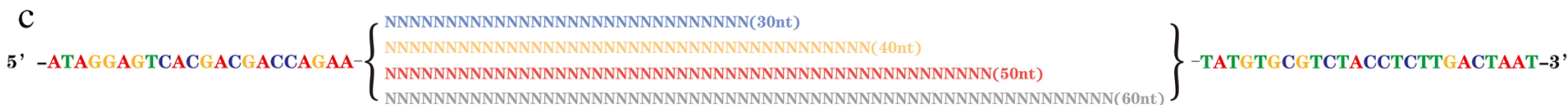

Fig. 1 Improved SELEX technology. a SDS-PAGE analysis of the coupling effect between magnetic beads and LDH protein; lane 1, magnetic bead supernatant coupled with normal human serum; lane 2, normal human serum dissociated from magnetic beads; lane 3, magnetic bead supernatant coupled with LDH protein; lane 4, LDH protein dissociated from the magnetic beads. b SELEX route. $\mathbf{c}$ Schematic diagram of the length-mixed library

\section{Affinity verification}

In order to determine the binding affinity of the nucleic acid aptamer library with the LDH protein in different rounds, the $5^{\prime}$ end of aptamer library was labeled with a fluorophore (Texas Red). After each round of incubation, fluorescence microscopy imaging was used to detect the binding intensity of the library with the LDH protein. As shown in Fig. 2a, no fluorescence was observed in the magnetic beads and LDH, indicating that the initial library cannot bind to LDH. After the first and second rounds of screening, the aptamers showed weak binding affinity to LDH protein. After the third round of screening, aptamers were seen to gradually bind to LDH, with obvious fluorescence observed in the 7th to 9th round libraries, indicating their strong binding ability. Wang $\mathrm{J}$ also used negative screening to select renal cell carcinoma (RCC) aptamers and obtained enriched sequences after 12 rounds of screening [31]. In the present study, from the third round onward, a more obvious enrichment signal appeared and reached the peak in the 7th round. A possible explanation is that the initial ssDNA library has a larger capacity and may have a richer secondary structure, thereby improving the screening efficiency. The fluorescence intensity analysis also showed a significant increase between rounds 2 and 3 as well as rounds 6 and 7, with a very strong intensity in rounds 7-9 and maximal intensity in round 7. Meanwhile, we calculated the proportion of the magnetic beads with fluorescence intensity greater than 30 . The calculation was consistent with the fluorescence intensity result, with the peak in the seventh round (Fig. 2b, c). For quantitative analysis of the fluorescence intensity, we used a fluorescence spectrophotometer to measure the fluorescence values of the aptamers dissociated from the initial, 7th, 8th, and 9th libraries. After standard curve determination and statistical analysis, the affinity was shown to be significantly higher in the SELEX-screened libraries than the initial library (Fig. 2d).

\section{High-throughput sequencing and analysis}

The aptamer libraries obtained from 9 rounds of screening were PCR amplified, followed by purification of target fragments by gel recovery, and then, the Illumina sequencing platform was used for high-throughput sequencing. First, the sequencing files were analyzed with selfedited software to obtain the proportion trend of aptamer libraries of different lengths during the 1-9 rounds of screening (Fig. 3a). Among them, N30 accounted for the largest proportion and gradually increased with the screening round, while N60 accounted for the lowest proportion and decreased continuously with the screening round. In the 1-9 rounds, N50 and N60 accounted for a stable proportion with small fluctuations, indicating that the secondary structure formed by $30-40$ nt ssDNA can more easily bind to LDH protein. The MEME website analysis of the motifs in the aptamer libraries of different rounds showed a very low degree of conservation in 


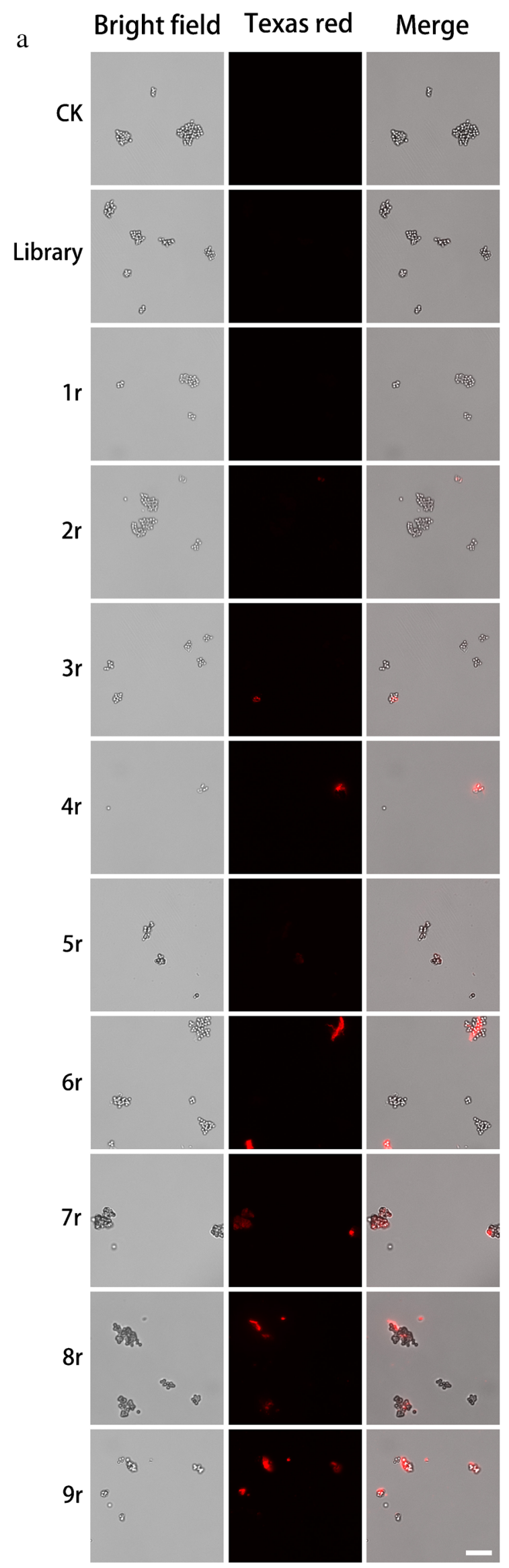

b

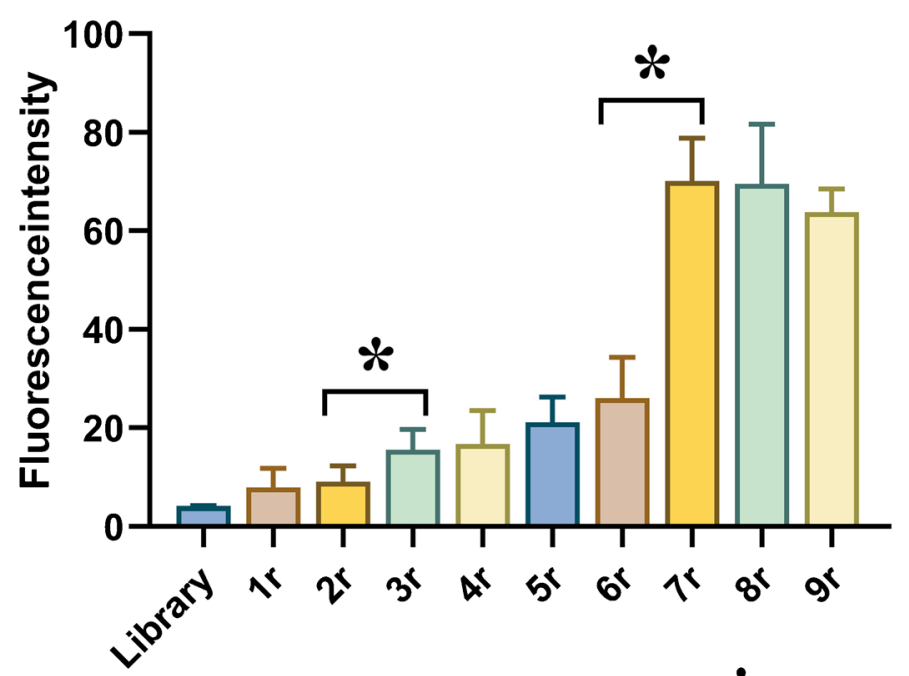

c

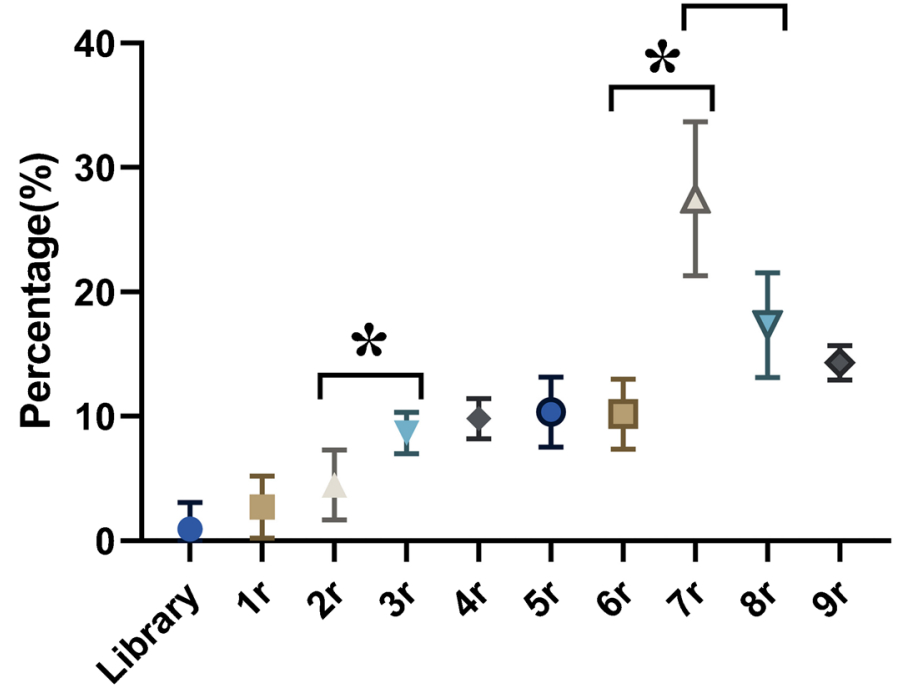

d

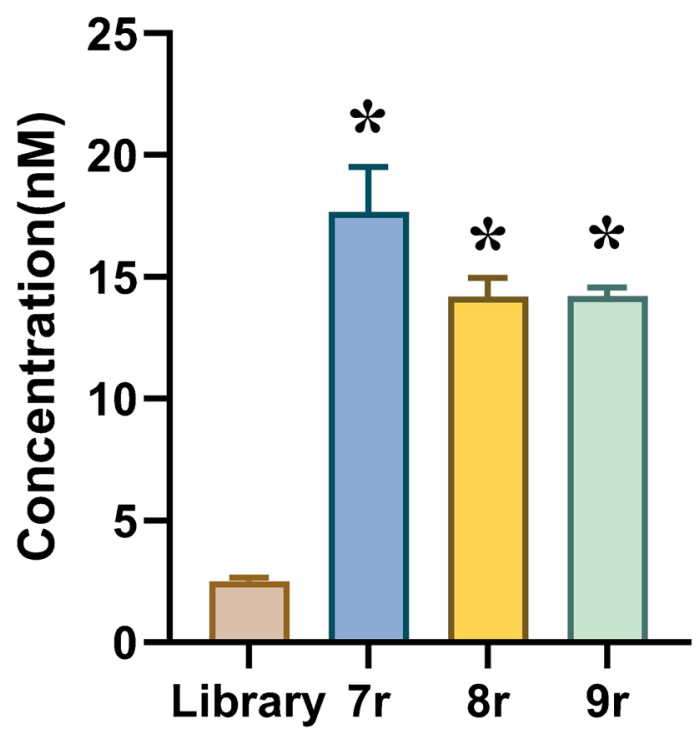


Fig. 2 Affinity verification. a Microscopic imaging for the LDH protein aptamer library in each of the 9 rounds. CK, a carboxyl magnetic bead coupled with $\mathrm{LDH}$ protein only, scale bar $=5 \mu \mathrm{m}$. b Microscope imaging fluorescence intensity statistics for the LDH protein aptamer library in each round. c The ratio of magnetic beads with fluorescence intensity greater than 30 in the microscope imaging for the LDH protein aptamer libraries in each round. $\mathbf{d}$ The fluorescence intensity in the initial, 7th, 8th, and 9th libraries, with the initial library used as a control. $* p<0.05$ rounds $1-3$, with conserved regions rich in $\mathrm{C}$ and $\mathrm{T}$ sequences. In rounds 4 and 5 , the degree of conservation increased, but the composition of the original conserved sequences was relatively unstable. The 6 th, 7 th, and 9 th round conservative elements appeared frequently, and they were all A- and G-rich sequences with a length of $21 \mathrm{nt}$. The length of the 8 th round was $15 \mathrm{nt}$, which

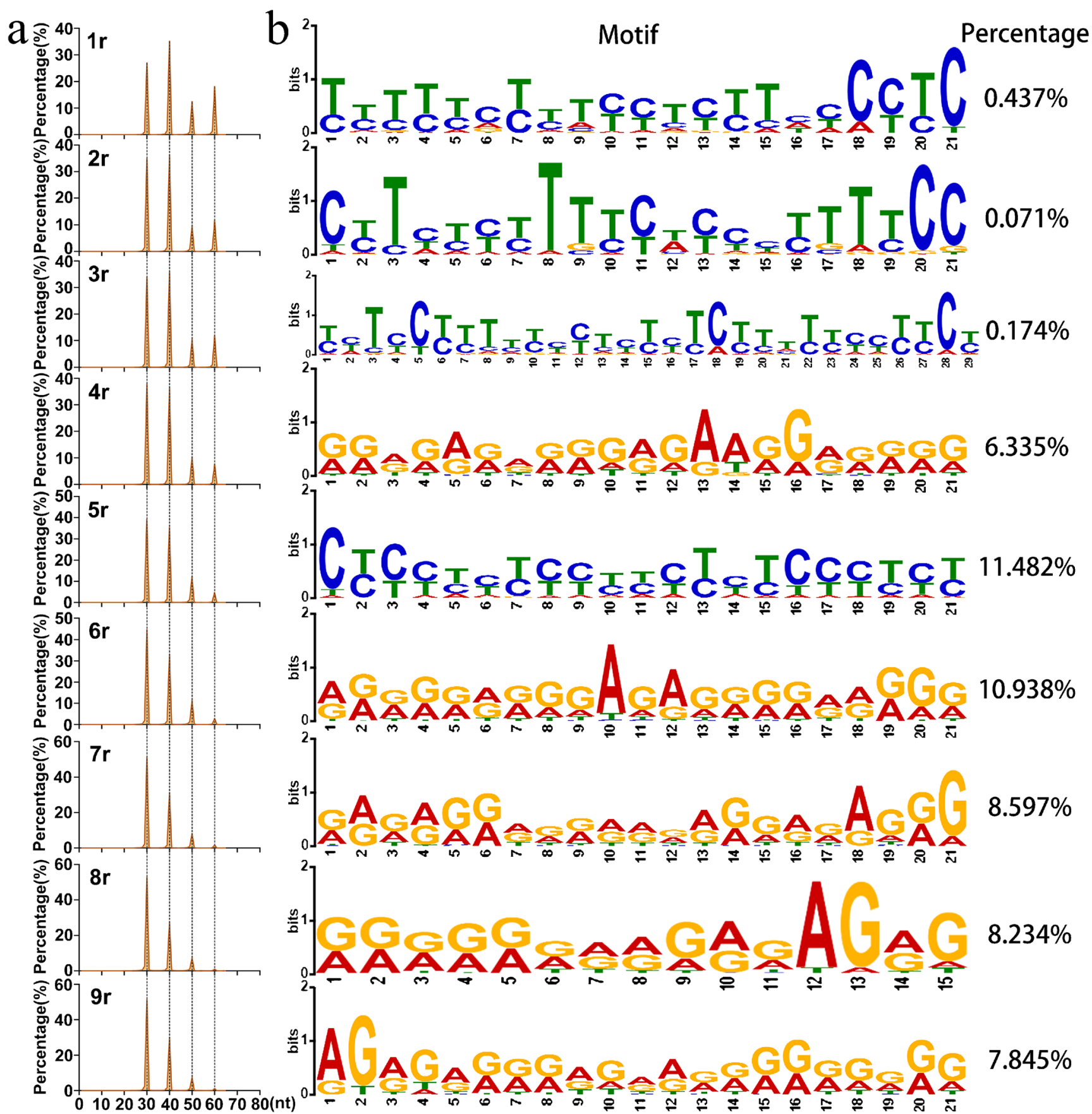

Fig. 3 High-throughput sequencing and bioinformatics analysis. a The proportion of aptamers of different lengths in the 1-9 aptamer libraries screened by SELEX. b Motifs and their percentages in the 1-9 aptamer libraries 
indicated that the single site had a greater effect on aptamer affinity relative to the sequence length. The 6th and 10th base $A$ in the 6th round library was highly conservative, with an obvious difference from the 7 th to 9 th round libraries (Fig. 3b).

The NUPACK website was used to predict the secondary structure of part of the highly conserved sequences selected in rounds 1 to 9 , and these sequences were seen to have relatively abundant stem-loop structures (Fig. 4), which provide sufficient sites for the specific binding of aptamer and LDH protein. Compared with clone sequencing, the sequences selected through high-throughput sequencing and bioinformatics analysis showed significantly higher enrichment in the secondary structure and conserved elements [32-34]. Based on the affinity results in different rounds of screening, candidate LDH protein aptamer sequences with higher affinity were selected from the 7th, 8th, and 9th round aptamer libraries to further verify their affinity and specificity. By comparing the A and $\mathrm{G}$ conservation degree of the sequences, we finally selected 15 sequences with high conservation degree and large difference in the secondary structure as candidate LDH protein aptamer sequences. Their free energies were all in the range of $-10 \sim-3 \mathrm{kcal} /$ mol to form stable secondary structures, and the information for these 15 sequences is listed in Table 2 .
Affinity analysis of LDH aptamer candidates

As shown by the fluorescence microscopy imaging in Fig. 5, the 15 selected aptamer candidate sequences can bind to LDH protein, but not to normal human serum, AFP, and BSA. Incubation of the 15 candidate sequences with normal human serum showed no red fluorescence in the microscope imaging, indicating significant negative screening effect. Under the same conditions, the fluorescently labeled aptamer candidate sequences were incubated with LDH protein. Obvious fluorescence was shown from LDH7-1, LDH7-5, LDH7-9, LDH8-2, LDH8-3, LDH9-1, and LDH9-2, whose coupling effect is better and fluorescence is stronger than that of other sequences based on the microscope imaging. Meanwhile, their specific binding to LDH protein was more stable, with fluorescent signals in almost all magnetic beads.

Furthermore, the microscope imaging data were semiquantified using ImageJ software. Statistical analysis showed significant difference among these 15 candidate sequences in their binding affinity to LDH protein and normal human serum, with a better performance for LDH7-1, LDH7-9, LDH8-2, and LDH9-1 than the other candidate sequences. Meanwhile, we calculated the percentage of magnetic beads

Table 215 candidate LDH aptamer sequences

\begin{tabular}{llcc}
\hline Aptamer & Sequences (5'-3') & $\begin{array}{c}\text { Length (nt) } \\
\text { Free energy } \\
(\mathrm{kcal} / \mathrm{mol})\end{array}$ \\
\hline LDH7r-1 & TTTCGGGATGATGGTTTTATGGCCGCTTCG & 30 & -3.47 \\
LDH7r-2 & GAGGTCCCGCATGAGAAAGCCCATCACCGC & 30 & -8.32 \\
LDH7r-3 & CATGATAGTTGGACTGGCAAGCTTGGGGGA & 30 & -4.03 \\
LDH7r-4 & CACGGTACCGATTGGCATGATAGTTCACAC & 30 & -5.42 \\
LDH7r-5 & AGGCTGATAGTTTGTGGGGGCTGTACAATG & 30 & -4.13 \\
LDH7r-6 & GGGCAAATAGCATAATGGATCACATTAGATGAGCCCAGGG & 40 & -7.20 \\
LDH7r-7 & CAGTTATGATAGTTGGCAAAGAGATGATGGTTGTGTTTGG & 40 & -2.76 \\
LDH7r-8 & CACCACACTTAGTTCGTCCCCGATGATAGTTTGCTGAGCA & 40 & -3.81 \\
LDH7r-9 & AGAGTGCTACAGCATGATGGTTTGGGCACCGTACCGAACG & 40 & -7.57 \\
LDH8r-1 & CGAGCTGAGATTGGGGAACTCGACGACAGTCAAGGGTCTG & 40 \\
LDH8r-2 & AGACTTCCGATAGGTTAGGGTTATAGAAAATGCAGGGGAGTAAAAAGAGC & 50 & -9.78 \\
LDH8r-3 & AGGGGAAAAAGGAGAGAACGAGAGAGAAGAGAGTATGGCAGACATGGCATG & 51 & -4.87 \\
LDH9r-1 & GCATGTGAGGAGAGAACAAATGAAGTGGCC & 30 \\
LDH9r-2 & GGGCCGATGTCACAGGTGAGACCATATTTTGCCTGGCCTG & 40 & -6.07 \\
LDH9r-3 & GGGATAGAGGAAAGAGAGATAGAGAGTCAGGGCAAGGGGTGAGAGGAGGG & 50 & -4.03 \\
\hline
\end{tabular}


with a fluorescence intensity greater than 50 , and the four sequences (LDH7-1, LDH7-9, LDH8-2, and LDH9-1) were also shown to have a significantly higher ratio, all higher than $60 \%$ (Fig. 6). To identify the affinity of candidate aptamers with the LDH protein, the method of fluorescence assay was used to determine the dissociation constant $\left(K_{d}\right)$ [21]. After kinetic curve fitting, the $K_{d}$ of LDH7r-1, LDH7r9, LDH8r-2, and LDH9r-1 are respectively $21.3 \pm 3.9 \mathrm{nM}$, $12.0 \pm 4.4 \mathrm{nM}, 10.2 \pm 4.7 \mathrm{nM}$, and $19.3 \pm 6.2 \mathrm{nM}$ (Fig. 7). These four candidate aptamer sequences all have strong affinity with LDH protein, and the binding amount is significantly higher compared with the initial library. These 4 aptamer sequences have the potential use for LDH content and enzyme activity detections, which could further combine with the microfluidic chip and surface-enhanced Raman spectroscopy to construct a high-efficiency and low-cost method for rapid disease diagnosis.

\section{Conclusion}

In this study, 9 rounds of aptamer screening were performed for the length-mixed library using the SELEX
Fig. 4 The secondary structure of typical aptamers in the 1 st -9 th round aptamer libraries. Triangle represents one of the 15 selected candidate LDH aptamer sequences
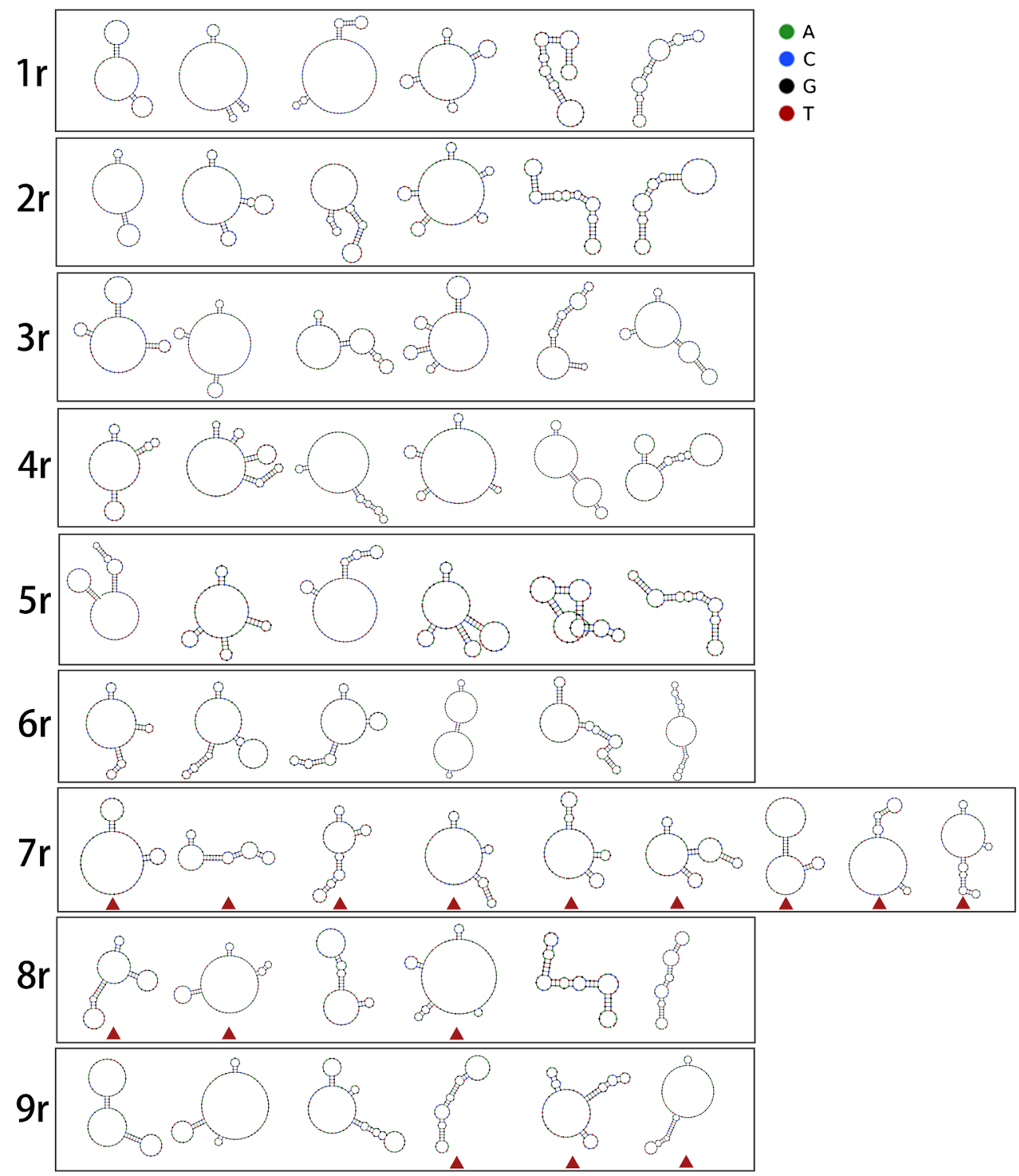

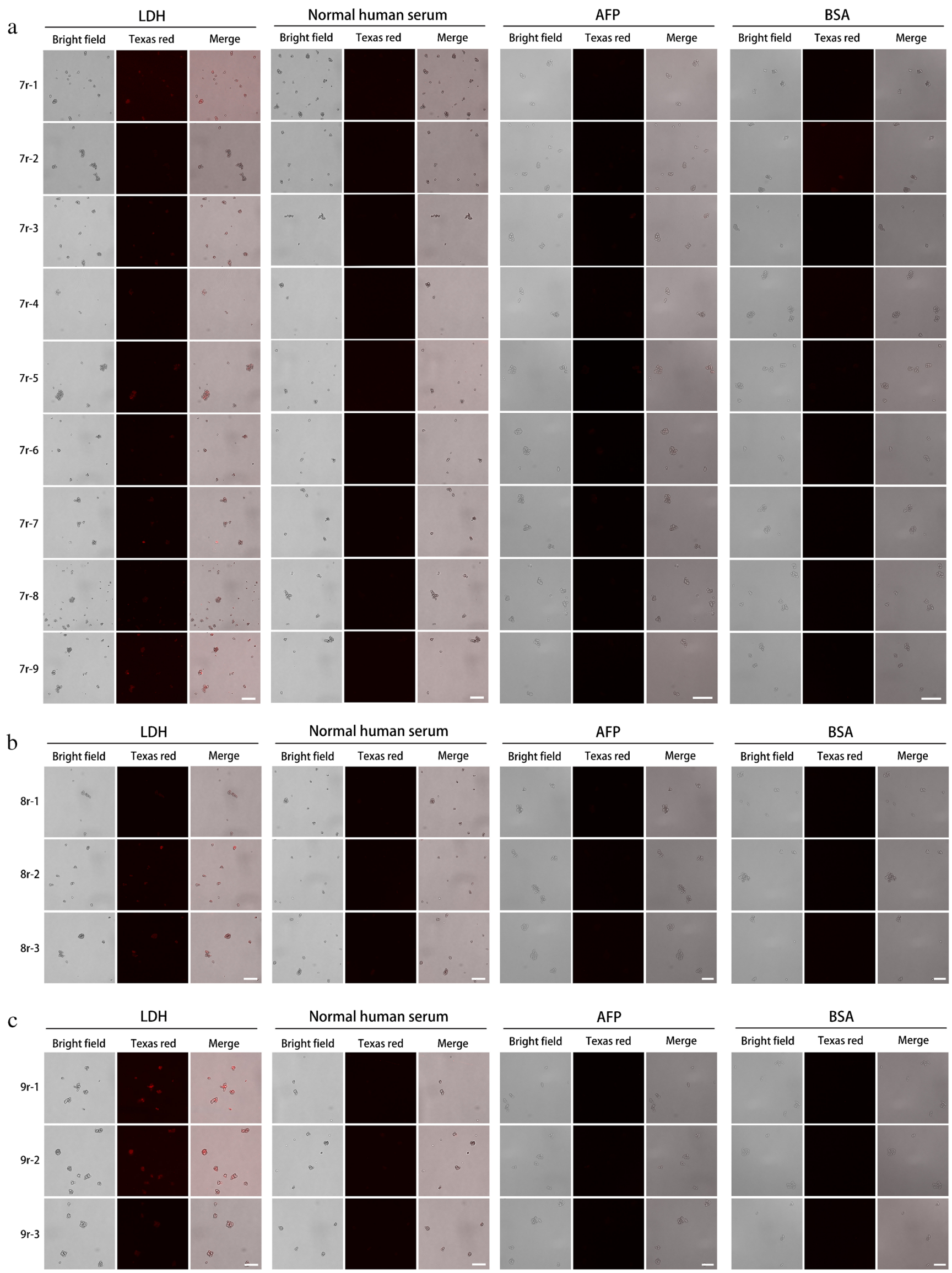
Fig. 5 Fluorescence microscope imaging of LDH protein aptamer candidates. a Candidate sequences from the 7 th and 9 th rounds of selection. b 3 candidate sequences from the 8 th round of selection. c 3 candidate sequences from the 9 th round of selection. Scale bars $=15 \mu \mathrm{m}$

Fig. 6 Statistical results for the fluorescence microscope imaging of candidate aptamers. a-c

Fluorescence intensity of $15 \mathrm{LDH}$ aptamer candidates. The controls: LDH in each aptamer candidate. d-f The percentage of LDHcoupled magnetic beads with a fluorescence intensity greater than 50 in the microscope imaging of $15 \mathrm{LDH}$ aptamer candidates. $* p<0.05$. The controls: $7 \mathrm{r}-1$ in panel d, $8 \mathrm{r}-2$ in panel $\mathbf{e}$, and $9 \mathrm{r}-1$ in panel $\mathbf{f}$
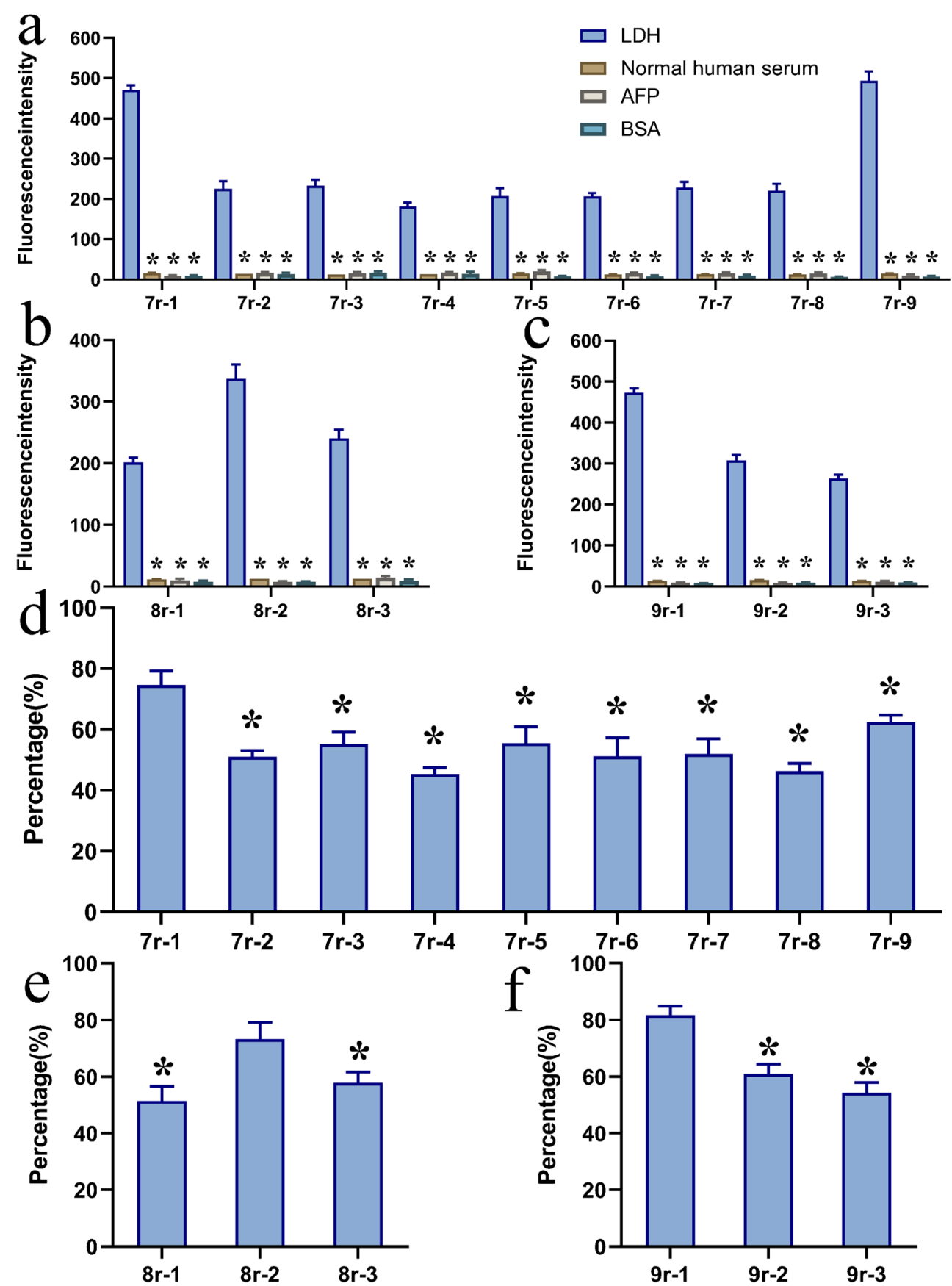

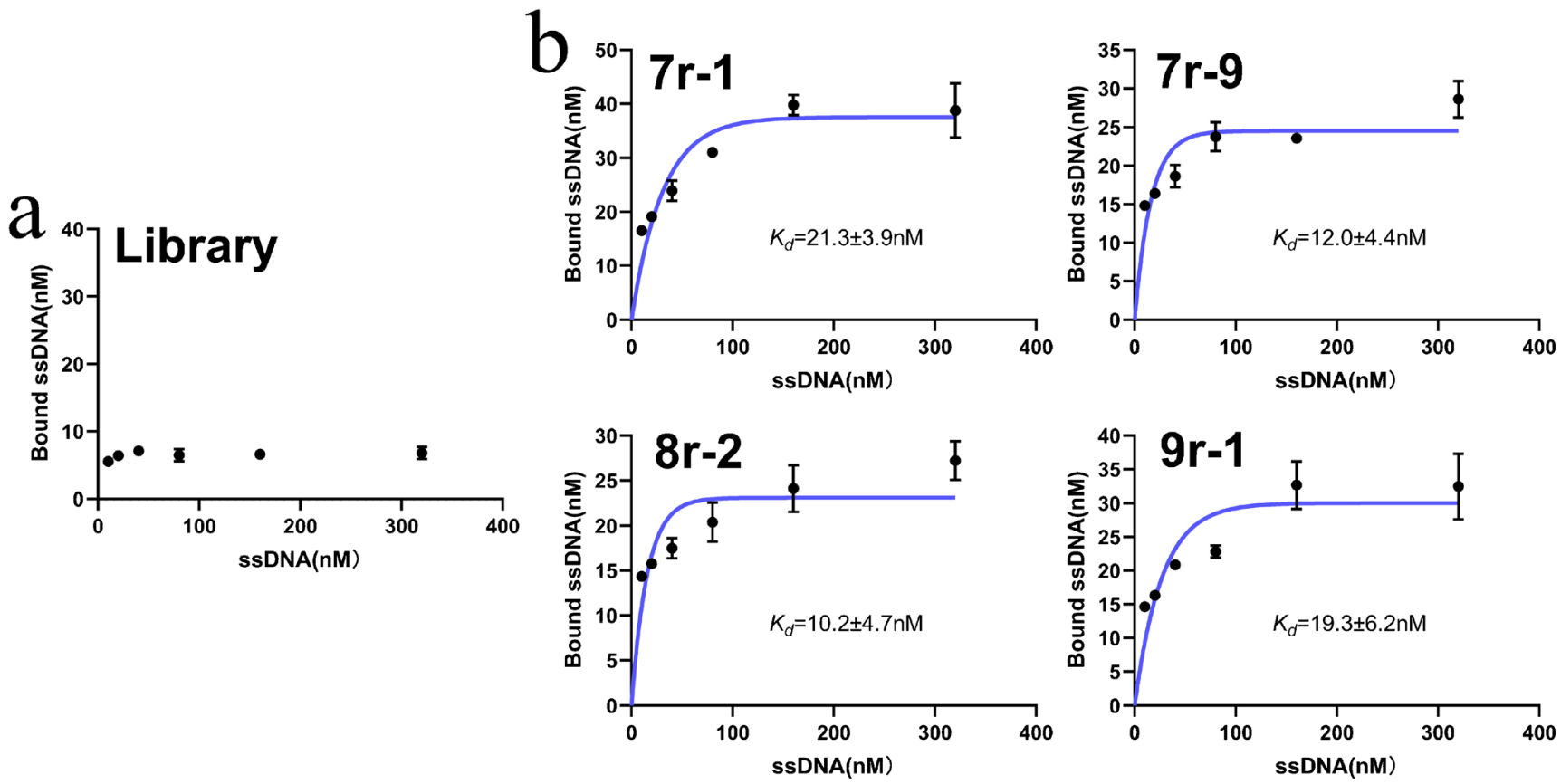

Fig. 7 Fluorescence intensity measurements to determine $K_{d}$ of $4 \mathrm{LDH}$ aptamer candidates. a Fluorescence intensity of the native library. b Fluorescence intensity of $4 \mathrm{LDH}$ aptamer candidates. The native

technology, and the affinity was significantly improved in the $3 \mathrm{rd}$ and 7 th rounds of selection. Through highthroughput sequencing, motif analysis, and secondary structure prediction, we finally selected 15 aptamer sequences in the 7 th -9 th rounds of screening, and four of them (LDH7-1, LDH7-9, LDH8-2, LDH9-1) were shown to bind to LDH protein with high affinity and specificity. This study laid an experimental foundation for the rapid detection of LDH protein content and enzyme activity and also provided new ideas for the rapid detection of other serum markers.

Funding This work was supported by National Undergraduate Training Programs for Innovation and Entrepreneurship (202010504032).

Declarations The authors declare no competing interests.

\section{References}

1. Augoff K, Grabowski K. Przydatność oznaczania dehydrogenazy mleczanowej w rozpoznawaniu chorób nowotworowych [Significance of lactate dehydrogenase measurements in diagnosis of malignancies]. Pol Merkur Lekarski. 2004;17(102):644-7.

2. Hsu PP, Sabatini DM. Cancer cell metabolism: Warburg and beyond. Cell. 2008;134(5):703-7.

3. Urbanska K, Orzechowski A. Unappreciated role of LDHA and LDHB to control apoptosis and autophagyin tumor cells. Int $\mathrm{J}$ Mol Sci. 2019;20:2085. library exhibits negligible binding to LDH protein while the $K_{d}$ of LDH7r-1, LDH7r-9, LDH8r-2, and LDH9r-1 are respectively $21.3 \pm$ $3.9 \mathrm{nM}, 12.0 \pm 4.4 \mathrm{nM}, 10.2 \pm 4.7 \mathrm{nM}$, and $19.3 \pm 6.2 \mathrm{nM}$

4. Jurisic V, Radenkovic S, Konjevic G. The actual role of LDH as tumor marker, biochemical and clinical aspects. Adv Exp Med Biol. 2015;867:115-24.

5. Gholizadeh N, Alipanahi Ramandi M, Motiee-Langroudi M, Jafari M, Sharouny H, Sheykhbahaei N. Serum and salivary levels of lactate dehydrogenase in oral squamous cell carcinoma, oral lichen planus and oral lichenoid reaction. BMC Oral Health. 2020;20(1): 314.

6. Ping W, Senyan H, Li G, Yan C, Long L. Increased lactate in gastric cancer tumor-infiltrating lymphocytes is related to impaired $\mathrm{T}$ cell function due to miR-34a deregulated lactate dehydrogenase A. Cell Physiol Biochem. 2018;49(2):828-36.

7. Mezquita L, Auclin E, Ferrara R, Charrier M, Remon J, Planchard $\mathrm{D}$, et al. Association of the Lung Immune Prognostic Index with immune checkpoint inhibitor outcomes in patients with advanced non-small cell lung cancer. JAMA Oncol. 2018;4(3):351-7.

8. Liu Y, Guo JZ, Liu Y, Wang K, Ding W, Wang H, et al. Nuclear lactate dehydrogenase A senses ROS to produce $\alpha$ hydroxybutyrate for HPV-induced cervical tumor growth. Nat Commun. 2018;9(1):4429.

9. Das CK, Parekh A, Parida PK, Bhutia SK, Mandal M. Lactate dehydrogenase A regulates autophagy and tamoxifen resistance in breast cancer. Biochim Biophys Acta, Mol Cell Res. 2019;1866(6): 1004-18.

10. Ding J, Karp JE, Emadi A. Elevated lactate dehydrogenase (LDH) can be a marker of immune suppression in cancer: interplay between hematologic and solid neoplastic clones and their microenvironments. Cancer Biomark. 2017;19(4):353-63.

11. Henry BM, Aggarwal G, Wong J, Benoit S, Vikse J, Plebani M, et al. Lactate dehydrogenase levels predict coronavirus disease 2019 (COVID-19) severity and mortality: a pooled analysis. Am J Emerg Med. 2020;38(9):1722-6.

12. Henry BM, Benoit SW, de Oliveira MHS, Hsieh WC, Benoit J, Ballout RA, et al. Laboratory abnormalities in children with mild and severe coronavirus disease 2019 (COVID-19): a pooled analysis and review. Clin Biochem. 2020;81:1-8. 
13. Ellington AD, Szostak JW. In vitro selection of RNA molecules that bind specific ligands. Nature. 1990;346(6287):818-22.

14. Sun H, Zhu X, Lu PY, Rosato RR, Tan W, Zu Y. Oligonucleotide aptamers: new tools for targeted cancer therapy. Mol Ther Nucleic Acids. 2014;3(8):e182.

15. Cruz-Aguado JA, Penner G. Determination of ochratoxin a with a DNA aptamer. J Agric Food Chem. 2008;56(22):10456-61.

16. Li WM, Bing T, Wei JY, Chen ZZ, Shangguan DH, Fang J. CellSELEX-based selection of aptamers that recognize distinct targets on metastatic colorectal cancer cells. Biomaterials. 2014;35(25): 6998-7007.

17. Ninomiya K, Kaneda K, Kawashima S, Miyachi Y, Ogino C, Shimizu N. Cell-SELEX based selection and characterization of DNA aptamer recognizing human hepatocarcinoma. Bioorg Med Chem Lett. 2013;23(6):1797-802.

18. Zhou W, Zhao L, Yuan H, Xu L, Tan W, Song Y, et al. A new small cell lung cancer biomarker identified by Cell-SELEX generated aptamers. Exp Cell Res. 2019;382(2):111478.

19. Chen C, Zhou S, Cai Y, Tang F. Nucleic acid aptamer application in diagnosis and therapy of colorectal cancer based on cell-SELEX technology. NPJ Precis Oncol. 2017;1(1):37.

20. Sinha A, Gopinathan P, Chung YD, Lin HY, Li KH, Ma HP, et al. An integrated microfluidic platform to perform uninterrupted SELEX cycles to screen affinity reagents specific to cardiovascular biomarkers. Biosens Bioelectron. 2018;122:104-12.

21. Cho M, Xiao Y, Nie J, Stewart R, Csordas AT, Oh SS, et al. Quantitative selection of DNA aptamers through microfluidic selection and high-throughput sequencing. Proc Natl Acad Sci U S A. 2010;107(35):15373-8.

22. Zhong Y, Zhao J, Li J, Liao X, Chen F. Advances of aptamers screened by Cell-SELEX in selection procedure, cancer diagnostics and therapeutics. Anal Biochem. 2020;598:113620.

23. Tan Y, Li Y, Yi-Xin Q, Yuanye S, Peng Y, Zhao Z, et al. Aptamerpeptide conjugates as targeted chemosensitizers for breast cancer treatment. ACS Appl Mater Interfaces. 2020. https://doi.org/10. 1021/acsami.0c18282.

24. Hao Y, Ren J, Liu C, Li H, Liu J, Yang Z, et al. Zinc protects human kidney cells from depleted uranium-induced apoptosis. Basic Clin Pharmacol Toxicol. 2014;114(3):271-80.
25. Kim JW, Kim EY, Kim SY, Byun SK, Lee D, Oh KJ, et al. Identification of DNA aptamers toward epithelial cell adhesion molecule via cell-SELEX. Mol Cell. 2014;37(10):742-6.

26. Bailey TL, Boden M, Buske FA, Frith M, Grant CE, Clementi L, et al. MEME SUITE: tools for motif discovery and searching. Nucleic Acids Res. 2009;37(Web Server issue):W202-8.

27. Zhong W, Pu Y, Tan W, Liu J, Liao J, Liu B, et al. Identification and application of an aptamer targeting papillary thyroid carcinoma using Tissue-SELEX. Anal Chem. 2019;91(13):8289-97.

28. Oh SS, Qian J, Lou X, Zhang Y, Xiao Y, Soh HT. Generation of highly specific aptamers via micromagnetic selection. Anal Chem. 2009;81(13):5490-5.

29. Mirian M, Kouhpayeh S, Shariati L, Boshtam M, Rahimmanesh I, Darzi L, et al. Generation of HBsAg DNA aptamer using modified cell-based SELEX strategy. Mol Biol Rep. 2021;48(1):139-46.

30. Ozer A, White BS, Lis JT, Shalloway D. Density-dependent cooperative non-specific binding in solid-phase SELEX affinity selection. Nucleic Acids Res. 2013;41(14):7167-75.

31. Wang J, Zhang Y, Chen Y, Hong S, Sun Y, Sun N, et al. In vitro selection of DNA aptamers against renal cell carcinoma using living cell-SELEX. Talanta. 2017;175:235-42.

32. Souza AG, Marangoni K, Fujimura PT, Alves PT, Silva MJ, Bastos VA, et al. 3D Cell-SELEX: development of RNA aptamers as molecular probes for PC-3 tumor cell line. Exp Cell Res. 2016;341(2): $147-56$.

33. Frith KA, Fogel R, Goldring JPD, Krause RGE, Khati M, Hoppe H, et al. Towards development of aptamers that specifically bind to lactate dehydrogenase of Plasmodium falciparum through epitopic targeting. Malar J. 2018;17(1):191.

34. Dupont DM, Larsen N, Jensen JK, Andreasen PA, Kjems J. Characterisation of aptamer-target interactions by branched selection and high-throughput sequencing of SELEX pools. Nucleic Acids Res. 2015;43(21):e139.

Publisher's note Springer Nature remains neutral with regard to jurisdictional claims in published maps and institutional affiliations. 